\title{
The association between dietary quality and well-being among middle-aged adults: a cross-sectional study
}

\author{
A.P. Meegan ${ }^{1}$ I.J. Perry ${ }^{2}$ and C.M. Phillips ${ }^{1,2}$ \\ ${ }^{1}$ HRB Centre for Diet and Health Research, School of Public Health, Physiotherapy and Sports Science, University \\ College Dublin, Ireland. and ${ }^{2} H R B$ Centre for Diet and Health Research, Dept. of Epidemiology and Public Health, \\ University College Cork, Ireland
}

More than a quarter of European adults have experienced a mental health disorder ${ }^{(1)}$. Several studies have demonstrated that certain micronutrients are associated with beneficial effects on mental health ${ }^{(2-3)}$. However, observing the effect of individual nutrients may not be representative of the impact of the habitual diet on mental health as nutrients are not consumed in isolation. Thus examination of the combined effects of multiple dietary components which reflect the whole diet may be preferable. Evidence regarding the psychological benefits associated with dietary quality and compliance with dietary guidelines is lacking. Therefore the aim of the present study is to examine potential associations between dietary composition, dietary quality and food pyramid compliance with mental health determined by depressive symptoms, anxiety and poor well-being.

Depressive symptoms, anxiety and well-being were assessed using the Centre for Epidemiologic Studies Depression Scale $(\text { CES-D })^{(4)}$, the Hospital Anxiety and Depression Scale (HADS) ${ }^{(5)}$ and the World Health Organization (WHO)-5 Well Being Index $^{(6)}$ in a cross-sectional sample of 2,047 Irish men and women aged 50 to 69 years. Diet was assessed using a self-completed food frequency questionnaire (FFQ). Macronutrient composition, daily servings from each food pyramid shelf, compliance with food pyramid recommendations and a dietary quality score (DASH (Dietary Approaches to Stop Hypertension) was calculated using the FFQ responses. Chi-square tests, t-tests and logistical regression analyses were used to investigate the associations between dietary components and mental health among the whole cohort and when stratified by gender and by BMI group.

\begin{tabular}{|c|c|c|c|c|c|c|}
\hline \multirow[b]{2}{*}{ Dietary Quality } & \multicolumn{2}{|c|}{ Depressive Symptoms } & \multicolumn{2}{|c|}{ Anxiety } & \multicolumn{2}{|c|}{ Poor well-being } \\
\hline & $\overline{\mathrm{OR}}(95 \% \mathrm{CI})$ & $\overline{p \text {-value }}$ & $\mathrm{OR}(95 \% \mathrm{CI})$ & $\overline{p \text {-value }}$ & $\overline{\mathrm{OR}}(95 \% \mathrm{CI})$ & $p$-value \\
\hline \multicolumn{7}{|l|}{ Model 1} \\
\hline Low & 1 [reference] & & 1 [reference] & & 1 [reference] & \\
\hline High & $0.84(0.63-1.13)$ & $0 \cdot 247$ & $0.83(0.61-1.14)$ & $0 \cdot 247$ & $0.62(0.47-0.82)$ & $0 \cdot 001$ \\
\hline \multicolumn{7}{|l|}{ Model 2} \\
\hline Low & 1 [reference] & & 1 [reference] & & 1 [reference] & \\
\hline High & $0.91(0.65-1.29)$ & 0.609 & $0.82(0.60-1.13)$ & $0 \cdot 218$ & $0.63(0.46-0.85)$ & $0 \cdot 002$ \\
\hline \multicolumn{7}{|l|}{ Model 3} \\
\hline Low & 1 [reference] & & 1 [reference] & & 1 [reference] & \\
\hline High & $1.06(0.69-1.63)$ & $0 \cdot 800$ & $0.77(0.52-1.16)$ & $0 \cdot 217$ & $0.60(0.41-0.87)$ & $0 \cdot 007$ \\
\hline
\end{tabular}

High dietary quality (above median) was associated with lower risk of poor well-being (OR $0 \cdot 60,95 \%$ CI $0 \cdot 41-0 \cdot 87, p=0 \cdot 007)$ relative to those with low dietary quality (below median). This association remained significant among females $(\mathrm{OR}=0 \cdot 52, \mathrm{CI}$ $0 \cdot 31-0.88, p=0.014)$ and non-obese individuals $(\mathrm{OR}=0.49,95 \% \mathrm{CI} 0.31-0.78, p=0.003)$. Although there was a trend towards reduced risk of depressive symptoms and anxiety among those with high dietary quality these findings did not reach statistical significance. Dietary composition and compliance with food pyramid recommendations were not associated with mental health outcomes. In conclusion, these novel results highlight the importance of dietary quality in maintaining optimal mental health. Better understanding of the relationship between dietary quality and mental health may provide insight into potential therapeutic or intervention strategies to improve well-being.

1. WHO (2015) Mental health: data and statistics. Geneva: WHO.

2. Pasco JA, Jacka FN, Williams LJ et al. (2012) Complement Ther Med 20, 119-23.

3. Vashum KP, McEvoy M, Milton AH et al. (2014) J Affect Disord 166, 249-57.

4. Radloff LS (1977) Appl Psychol Meas 1, 385-401.

5. Zigmond AS, Snaith RP (1983) Acta Psychiatr Scand 67, 361-370.

6. WHO (1998) WHO (Five) Well-Being Index (1998 version). Geneva: WHO. 